DSF-T-93/21

hep-th/9308026

\title{
Dynamical Aspects of Lie-Poisson Structures
}

\author{
F. LIZZI, G. MARMO, G. SPARANO and P. VITALE"' \\ Dipartimento di Scienze Fisiche and I.N.F.N., Sez. di Napoli \\ Mostra d'Oltremare Pad. 19, 80125 Napoli.
}

\begin{abstract}
Quantum Groups can be constructed by applying the quantization by deformation procedure to Lie groups endowed with a suitable Poisson bracket. Here we try to develop an understanding of these structures by investigating dynamical systems which are associated with this bracket. We look at $S U(2)$ and $S U(1,1)$, as submanifolds of a 4-dimensional phase space with constraints, and deal with two classes of problems. In the first set of examples we consider some hamiltonian systems associated with Lie-Poisson structures and we investigate the equations of the motion. In the second set of examples we consider systems which preserve the chosen bracket, but are dissipative. However in this approach, they survive the quantization procedure.
\end{abstract}

May 1993

\footnotetext{
*LIZZI@NA.INFN.IT, GIMARMO@NA.INFN.IT, SPARANO@NA.INFN.IT, VITALE@NA.INFN.IT
} 


\section{Introduction}

The presence of a Poisson bracket on a manifold is an important ingredient to start the quantization procedure of dynamical systems. When the manifold is a Lie Group, of particular interest are Lie-Poisson brackets. In this case these Lie groups are called Lie-Poisson groups. The aim of this paper is to consider Lie-Poisson groups as carrier spaces of dynamical systems. The interest for LiePoisson groups stems from the observation that they may be regarded as "dequantization" of Quantum Groups; we hope thereof to gain an understanding of these structures by putting them into a dynamical perspective.

As it is well known quantum groups can be seen as non commutative generalizations of topological spaces which have a group structure; such a structure induces an abelian Hopf algebra structure on the algebra of smooth functions $\mathcal{F}(G)$ defined on the group. Quantum groups are defined then as non abelian Hopf algebras ( [1, 2]). A way to generate them consists in deforming the product of the abelian Hopf algebra of functions $\mathcal{F}(G)$ into a nonabelian one (*-product), using the so called quantization by deformation procedure or $*$-quantization. ([1, 2, 3]). This quantization technique gives a deformed product once it is assigned a Poisson bracket on the algebra $\mathcal{F}(G)$. In order to obtain that the deformed algebra is a Hopf algebra, namely a quantum group, the starting group $G$ has to be endowed with a Lie-Poisson structure, that is, the group multiplication, $m: G \times G \rightarrow G$, must be a Poisson map. Lie groups which enjoy this property are said Lie-Poisson groups. Being our interest mainly concerned with exemplification, we refer to [11, 2, 4] for the general theory.

We will consider two examples of three dimensional Lie groups, namely $S U(2)$ and $S U(1,1)$, as carrier spaces of dynamical systems, and we will look at the group manifold as a 3-dimensional surface in $\mathbb{R}^{4}$. We will then consider a Lie-Poisson structure on $G$, that is a bivector field $\Lambda$ which defines a Poisson bracket on the $\mathcal{F}(G)$ algebra of smooth functions on $G$

$$
\Lambda(d f \wedge d g)=\{f, g\} \quad f, g \in \mathcal{F}(G)
$$

in addition, such a bracket is also compatible with the group structure, being the group multiplication a Poisson map. Since the group manifold is three dimensional, the Poisson bracket must be degenerate, that is there must be, at least 
locally, a Casimir function $\mathcal{C}_{1} \in \mathcal{F}(G)$ such that:

$$
\left\{\mathcal{C}_{1}, f\right\}=0 \quad \forall f \in \mathcal{F}(G)
$$

Moreover, when we regard the group $G$ as a three dimensional surface in $\mathbb{R}^{4}$, vector fields on $\mathbb{R}^{4}$ entering in the definition of $\Lambda$, must be tangent to the group submanifold. If this submanifold is defined by the level set of a function $\mathcal{C}_{2}$, this function will be another Casimir for our bracket.

Lie-Poisson structures which we will consider on $S U(2)$ and $S U(1,1)$ are of the form

$$
\Lambda=r^{i j}\left(X_{i} \wedge X_{j}-\tilde{X}_{i} \wedge \tilde{X}_{j}\right)
$$

with $r^{i j} \in \mathbb{R}, X_{i}$ the left invariant vector fields, and $\tilde{X}_{j}$ the right invariant ones. In fact for simple Lie groups such a structure not only satisfies the request of being Lie-Poisson, but all Lie-Poisson structures are of this form (《4).

\section{Hamiltonian Systems}

We now consider the Lie-Poisson structure defined in equation (3) in some specific cases, and investigate the dynamics one obtains in the presence of a Hamiltonian. Obviously there is great arbitrariness in the choice of the Hamiltonian, and in our examples we have chosen some 'natural' ones.

\section{$2.1 S U(2)$}

We indicate with $y^{\mu}, \mu=0, \ldots, 3$ some coordinates in $\mathbb{R}^{4}$ and represent $S U(2)$ as unitary $2 \times 2$ complex matrices of the form

$$
g=y^{0} \mathbb{I}+i \sigma_{j} y^{j}
$$

where the $\sigma$ 's are the Pauli matrices and $\mathbb{I}$ is the identity matrix. The group manifold is defined by the constraint:

$$
\operatorname{det} g=y_{0}^{2}+y_{1}^{2}+y_{2}^{2}+y_{3}^{2}=1 \text {. }
$$

In order to write the Poisson bracket (3) in $\mathbb{R}^{4}$ we use the following realization for left and right invariant vector fields:

$$
\begin{aligned}
X_{i} & =y^{0} \partial_{i}-y^{i} \partial_{0}+\varepsilon_{i j k} y^{j} \partial_{k} \\
\tilde{X}_{i} & =y^{0} \partial_{i}-y^{i} \partial_{0}-\varepsilon_{i j k} y^{j} \partial_{k} .
\end{aligned}
$$


Since the manifold is a 3-sphere all directions are equivalent and therefore we may take, without loss of generality, the Lie-Poisson bivector field to be

$$
\Lambda=\frac{1}{2}\left(X_{1} \wedge X_{2}-\tilde{X}_{1} \wedge \tilde{X}_{2}\right)
$$

Using the $\mathbb{R}^{4}$ representation of the vector fields given by (6) we may write the bivector field (7) in the following way:

$$
\Lambda=\left(y_{3} \partial_{0}-y_{0} \partial_{3}\right) \wedge y_{\mu} \partial_{\mu}+\operatorname{det} g \partial_{0} \wedge \partial_{3}
$$

with

$$
\operatorname{det} g=\mathcal{C}_{2}=y_{\mu} y^{\mu} \text {. }
$$

This function is in fact one of the two Casimirs of the Poisson bracket given by $\Lambda$. The other Casimir can easily be recognised to be any function of the ratio $\mathcal{C}_{1}=y_{2} / y_{1}$; for future convenience we will also consider the Casimir one-form related to $\mathcal{C}_{1}, \alpha_{1}$, defined as

$$
\alpha_{1}=y_{2} d y_{1}-y_{1} d y_{2}=-y_{1}^{2} d \mathcal{C}_{1}
$$

A 1 -form $\alpha$ is said to be a Casimir 1-form for $\Lambda$ if it admits an integrating factor and $\Lambda(\alpha)=0$. Under some regularity assumptions, Casimir 1-forms define codimension one submanifolds in the obvious way. The advantage of Casimir 1-forms with respect to Casimir functions is relevant when dealing with global properties. For instance $\alpha_{1}$ in (10) is smooth and well defined over all $\mathbb{R}^{2}$ while for $\mathcal{C}_{1}=y_{2} / y_{1}$ we have to remove $y_{1}=0$ thus getting a disconnected manifold $(\mathbb{R}-\{0\}) \times \mathbb{R}$. The Casimirs $\mathcal{C}_{2}$ and $\alpha_{1}$ define a two-dimensional surface, say $\Sigma$, where $\Lambda$ is non degenerate.

In order to consider a dynamics on $\Sigma$, let us make the following identifications:

$$
\left\{\begin{array} { l } 
{ y _ { 0 } = q _ { 2 } } \\
{ y _ { 3 } = p _ { 2 } }
\end{array} \quad \left\{\begin{array}{l}
y_{1}=q_{1} \\
y_{2}=p_{1}
\end{array}\right.\right.
$$

let us also define

$$
\begin{aligned}
H_{i} & =p_{i}^{2}+q_{i}^{2} \\
H & =H_{1}+H_{2}
\end{aligned}
$$

it follows that $\mathcal{C}_{2}=H$. Moreover, using the fact that bivector fields defined on a 2-dimensional surface can, at least locally, be written as $\Lambda=X \wedge Y$, the Poisson bivector field given by equation (8) may be expressed as:

$$
\Lambda=\left(\Delta-\frac{H}{H_{2}} \Delta_{2}\right) \wedge \Gamma_{2}
$$


where $\Delta$ is the dilation vector field in $\mathbb{R}^{4}$ written as:

$$
\begin{aligned}
\Delta & =\Delta_{1}+\Delta_{2} \\
\Delta_{i} & =q_{i} \partial_{q_{i}}+p_{i} \partial_{p_{i}} \quad i=1,2
\end{aligned}
$$

and $\Gamma_{2}$ is defined as:i]

$$
\Gamma_{2}=p_{2} \partial_{q_{2}}-q_{2} \partial_{p_{2}}
$$

Notice that decomposition (13) is not unique, in fact $\Lambda$ may be written as $\Lambda=\left(a_{1} X+b_{1} Y\right) \wedge\left(a_{2} X+b_{2} Y\right)$, with

$$
\begin{aligned}
& L_{X} \mathcal{C}_{1}=L_{X} \mathcal{C}_{2}=0 \\
& L_{Y} \mathcal{C}_{1}=L_{Y} \mathcal{C}_{2}=0
\end{aligned}
$$

and

$$
X \wedge Y \neq 0, \quad a_{1} b_{2}-a_{2} b_{1}=1
$$

so that there is a 3-parameter family of different decompositions of the bivector field (13).

Identifications (11), (12), suggest to regard $H_{1}$ and $H_{2}$ as oscillator Energies. We can use our bracket to associate with them equations of motion, the resulting system is composed by two non interacting oscillators, let us say 1 and 2 , whose total energy is fixed by the Casimir $\mathcal{C}_{2}$. Moreover the Casimir 1-form $\alpha_{1}$ may be recognized to be the differential of $\phi_{1}$, the phase of oscillator 1 , up to a multiplicative factor: $\alpha_{1}=p_{1} d q_{1}-q_{1} d p_{1}=H_{1} d \phi_{1}$. The equations of the motion are, for oscillator 2

$$
\begin{aligned}
\dot{q}_{2}=\left\{q_{2}, H_{2}\right\} & =-2 H_{1} p_{2} \\
\dot{p}_{2}=\left\{p_{2}, H_{2}\right\} & =2 H_{1} q_{2} \\
\left\{q_{1}, H_{2}\right\}=\left\{p_{1}, H_{2}\right\} & =0
\end{aligned}
$$

and for oscillator 1

$$
\begin{aligned}
\dot{q}_{1}=\left\{q_{1}, H_{1}\right\} & =0 \\
\dot{p}_{1}=\left\{p_{1}, H_{1}\right\} & =0 \\
\left\{q_{2}, H_{1}\right\}=\left\{p_{2}, H_{1}\right\} & =0
\end{aligned} .
$$

As we may observe $p_{1}$ and $q_{1}$ are 'frozen' degrees of freedom having vanishing Poisson brackets with $H_{1}$ and $H_{2}$, so that oscillator 1 does not evolve, but only

\footnotetext{
${ }^{\dagger}$ This vector field may be recognised as the one of a 1-dimensional harmonic oscillator.
} 
furnishes the parameter $H_{1}$, which is in fact a constant of the motion for oscillator 2. We therefore have a family of 1-dimensional oscillators whose motion is determined by an external parameter. We also observe that, since $H_{1}=H-H_{2}$ and $H$ is a constant, the system can also be regarded as a non-linear oscillator, in the sense that it has an energy dependent frequency, these kind of oscillators have being considered in the quantum group context [5].

We may conclude that, although the original phase space is 4-dimensional, the motion happens to be on a 2-dimensional surface, that is the dynamical system described has one degree of freedom, and its reduced phase space is defined by $\mathcal{C}_{1}$ and $\mathcal{C}_{2}$. Indeed it turns out to be an oscillator (the one we indicated as oscillator 2) which can be regarded either as a non linear one, (in the specified acception) or as a parameter dependent oscillator, the parameter being furnished by a reference oscillator (in our notation oscillator 1); we could say in the second case that the phase of one oscillator 'controls' the other. Therefore we learn that Poisson manifolds provide an appropriate setting to deal with parameter depending systems, each system leaving on a symplectic leaf of the given Poisson manifold.

\section{$2.2 S U(1,1)$}

In analogy with what has been done for $S U(2)$ we now consider a Lie-Poisson structure on $S U(1,1)$ and look for a dynamical system defined on the group. We represent the elements of the group as complex matrices with unit determinant:

$$
g=y^{0} \mathbb{I}+i \tilde{\sigma}_{j} y^{j}
$$

where $\mathbb{I}$ is the identity matrix and

$$
\tau_{i}=i \tilde{\sigma}_{i}
$$

are the infinitesimal generators of the group, the $\tilde{\sigma}_{i}$ 's being defined as:

$$
\tilde{\sigma}_{1}=\left(\begin{array}{cc}
0 & -i \\
-i & 0
\end{array}\right) \quad, \quad \tilde{\sigma}_{2}=\left(\begin{array}{cc}
0 & 1 \\
-1 & 0
\end{array}\right) \quad, \quad \tilde{\sigma}_{3}=\left(\begin{array}{cc}
1 & 0 \\
0 & -1
\end{array}\right)
$$

moreover $\operatorname{det} g=y_{0}^{2}-y_{1}^{2}-y_{2}^{2}+y_{3}^{2}=1$. The left and right invariant vector fields are respectively

$$
\begin{aligned}
& Y_{1 \pm}=y_{0} \partial_{1}+y_{1} \partial_{0} \pm\left(y_{2} \partial_{3}+y_{3} \partial_{2}\right) \\
& Y_{2 \pm}=y_{0} \partial_{2}+y_{2} \partial_{0} \mp\left(y_{3} \partial_{1}+y_{1} \partial_{3}\right) \\
& Y_{3 \pm}=y_{0} \partial_{3}-y_{3} \partial_{0} \mp\left(y_{1} \partial_{2}-y_{2} \partial_{1}\right)
\end{aligned}
$$


where $X_{i}=Y_{i+}, \quad \tilde{X}_{i}=Y_{i-}$. Also in this case we consider Poisson structures of the form

$$
\Lambda=X_{i} \wedge X_{j}-\tilde{X}_{i} \wedge \tilde{X}_{j}
$$

which are in fact Lie-Poisson structures, as already noted. From a topological point of view the group manifold is a connected hyperbolic surface in $\mathbb{R}^{4}$, that is an hypercilinder $S^{1} \times \mathbb{R}^{2}$. For this reason the various bivector-fields (21) that we may consider on $S U(1,1)$ are not equivalent. Basically, we have two inequivalent situations: one characterised by taking the two 'boosts', which in our notation are $X_{1}$ and $X_{2}\left(\tilde{X}_{1}, \tilde{X}_{2}\right.$, in the right invariant realization), the other characterised by taking the rotation vector field, namely $X_{3}$ and its right invariant partner, $\tilde{X}_{3}$, and one of the two 'boosts'.

\section{a) The 'boost-boost' case}

Let us first consider the case where the vector fields involved in the definition of the Poisson structure on $S U(1,1)$ are the two 'boosts'. In that case the bivector field is:

$$
\Lambda=\frac{1}{2}\left(X_{1} \wedge X_{2}-\tilde{X}_{1} \wedge \tilde{X}_{2}\right)
$$

Using the $\mathbb{R}^{4}$ representation of the vector fields given by (20) we have:

$$
\Lambda=-\left(y_{3} \partial_{0}-y_{0} \partial_{3}\right) \wedge \tilde{\Delta}-(\operatorname{det} g) \partial_{0} \wedge \partial_{3}
$$

where we have indicated with $\tilde{\Delta}$ the vector field:

$$
\tilde{\Delta}=y_{0} \partial_{y_{0}}+y_{3} \partial_{y_{3}}-y_{1} \partial_{y_{1}}-y_{2} \partial_{y_{2}}
$$

This Poisson structure is degenerate, having two Casimirs:

$$
\begin{gathered}
\mathcal{C}_{1}=\frac{y_{1}}{y_{2}} \\
\mathcal{C}_{2}=\operatorname{det} g .
\end{gathered}
$$

If we do the same identification of variables as in the previous example we obtain

$$
\mathcal{C}_{2}=H=H_{2}-H_{1}
$$

and the other Casimir is unchanged. We may decompose the bivector field $\Lambda$ the same way as before, that is

$$
\Lambda=\left(\Delta-\frac{H}{H_{2}} \Delta_{2}\right) \wedge \Gamma_{2}
$$


with $\Gamma_{2}, \Delta, \Delta_{i}$, having the same meaning as in the previous example. In fact the vector fields $X=\Delta-\frac{H}{H_{2}} \Delta_{2}$ and $Y=\Gamma_{2}$ still satisfy $L_{X} \mathcal{C}_{i}=L_{Y} \mathcal{C}_{i}=0$ with $i=1,2$. Let us look now at the equations of the motion in order to understand which kind of dynamical system we are describing. We have:

$$
\begin{aligned}
\dot{q}_{2} & =\left\{q_{2}, H_{2}\right\}=2 H_{1} p_{2} \\
\dot{p}_{2} & =\left\{p_{2}, H_{2}\right\}=-2 H_{1} q_{2} \\
\left\{q_{1}, H_{2}\right\} & =\left\{p_{1}, H_{2}\right\}=\left\{H_{1}, H_{2}\right\}=0 \\
\dot{q}_{1} & =\left\{q_{1}, H_{1}\right\}=0 \\
\dot{p}_{1} & =\left\{p_{1}, H_{1}\right\}=0 .
\end{aligned}
$$

Again, the system we are describing appears to be a pair of non interacting oscillators (being $\left\{H_{1}, H_{2}\right\}=0$ ), one of the two not evolving, so that the reduced system turns out to be a parameter dependent 1-dimensional oscillator, or a non linear one, depending on the possibility of writing $H_{1}$ as $H-H_{2}$. That is, we have obtained the same result as in the $S U(2)$ case. This result is due to the fact that the reduced phase space is, even in this case, a circle in the plane identified by coordinates $p_{2}$ and $q_{2}$. In fact the bivector (27) has a local Casimir $\mathcal{C}_{\infty}$, whose Casimir 1-form $\alpha_{1}=y_{2} d y_{1}-y_{1} d y_{2}$ may be written as:

$$
\alpha_{1}=p_{1} d q_{1}-q_{1} d p_{1} .
$$

This one-form defines a non degenerate surface (provided the other Casimir is imposed) in the plane $\left(p_{2}, q_{2}\right)$. In this plane the reduced phase space is defined by

$$
p_{2}^{2}+q_{2}^{2}=H+H_{1}
$$

which is a circle in the $\left(p_{2}, q_{2}\right)$ plane.

This result should not be a surprise, for we know that a given dynamical system may admit more than one Hamiltonian description. We are finding here that this statement may be true also for parameter depending systems.

\section{b) The 'rotation-boost' case}

As already noted, there is essentially only another inequivalent bivector field we may consider on $S U(1,1)$ which we expect to give rise to a different situation: namely we choose as starting vector fields a 'boost' and the rotation. For instance

$$
\Lambda=\frac{1}{2}\left(X_{2} \wedge X_{3}-\tilde{X}_{2} \wedge \tilde{X}_{3}\right)
$$


(an equivalent possibility is $\Lambda=\frac{1}{2}\left(X_{1} \wedge X_{3}-\tilde{X}_{1} \wedge \tilde{X}_{3}\right)$ ). We can still write $\Lambda$ in the form:

$$
\Lambda=\left(\Delta-\frac{H}{H_{2}} \Delta_{2}\right) \wedge \Gamma_{2}
$$

but we change the identification of the physical variables. We choose:

$$
\begin{array}{ll}
y_{2}=q_{1} & y_{1}=q_{2} \\
y_{3}=p_{1} & y_{0}=p_{2}
\end{array}
$$

With this choice

$$
\mathcal{C}_{2}=H=p_{2}^{2}+p_{1}^{2}-q_{2}^{2}-q_{1}^{2}=H_{1}+H_{2}
$$

where

$$
\begin{aligned}
& H_{1}=p_{1}^{2}-q_{1}^{2} \\
& H_{2}=p_{2}^{2}-q_{2}^{2}
\end{aligned}
$$

The previous identification is suggested by the Casimir 1-form $\alpha_{1}$ which in this case is:

$$
\alpha_{1}=y_{3} d y_{2}-y_{2} d y_{3}
$$

The expression of the Casimir suggests that $y_{2}$ and $y_{3}$ should be conjugate variables if we want to continue to think of $\mathcal{C}_{1}$ as a quantity which inherits only one of two particles constituting our dynamical system. Obviously other identifications will describe different dynamics and there is no preferred choice.

We observe that the hamiltonians $H_{1}, H_{2}$, are hamiltonians of 'inverted' oscillators, that is particles rolling down on inverted parabolic slope. As before, one 'oscillator' is controlled by the other, so that the trajectory of the reduced system in the phase space will be an hyperbola. This can be understood by the fact that $H_{1}$ (the Hamiltonian of the control system) is a constant of the motion for the system 2 , so, considering the Casimir $\mathcal{C}_{2}$, that is the total hamiltonian, we are left with:

$$
p_{2}^{2}-q_{2}^{2}=H-H_{1}
$$

the equation of an hyperbola. However, as in the previous examples, the 'frequency' of the inverted oscillator depends on the energy of the other one.

\section{Lie-Poisson Structures and Dissipative Fields}

The Lie Poisson structures we have considered, being constant combinations of left and right invariant bivector fields, belong to the class of quadratic Poisson brackets 
of $\mathbb{R}^{4}$, when we describe the 3- dimensional group manifold under consideration as embedded in $\mathbb{R}^{4}$. They are of the form

$$
\left\{y_{i}, y_{j}\right\}=R_{i j}^{r s} y_{r} y_{s}
$$

with $R_{i j}^{r s} \in \mathbb{R}$ and $y_{i}, i=0, . .3$ coordinates in $\mathbb{R}^{4}$. We want to consider now some particular aspects of quadratic Poisson brackets. The Poisson bracket (39) is left invariant by the dilation vector field

$$
\Delta=y_{j} \frac{\partial}{\partial y_{j}} .
$$

$\Delta$ can be thought of as a prototype of dissipative field, and thus, as dynamical vector field, is compatible with the Poisson bracket. On the other hand a quadratic Poisson Bracket, being zero along the zero of the quadratic form $R_{i j}^{r s} y_{r} y_{s}$, cannot be inverse of a symplectic structure. Therefore any quantization procedure relying on the existence of a symplectic structure cannot be applied in the present situation. However, since the deformed product is itself invariant under $\Delta$, at least for the two Lie groups we have considered [1], *-quantization procedure can still be used. So, in these cases, dissipative dynamical systems survive the transition to quantum groups, or they survive the quantization procedure. In the coming examples we will consider some dynamical systems preserving the Lie-Poisson group structure.

The form of the Poisson bivector fields considered until now, namely

$$
\Lambda=\left(\Delta-\frac{H}{H_{2}} \Delta_{2}\right) \wedge \Gamma_{2}
$$

suggests the investigation of dynamical fields of the form

$$
\Gamma=\frac{H}{H_{2}} \Delta_{2}-\Delta
$$

These vector fields, are compatible with the Lie-Poisson structures under consideration, that is

$$
L_{\Gamma} \Lambda=0
$$

so we are in the case we discussed above. As we have already mentioned, these dynamical fields may be automorphisms of the deformed (by *-quantization) algebra of observables, depending on whether or not the deformed product is a function of the Poisson bracket. Let us explain better this point. The commutator defined via the $*_{\hbar}$-product $\left(*_{\hbar}\right.$ means that the $*$ may be written as a series of powers in $\hbar)$

$$
[f, g]=f *_{\hbar} g-g *_{\hbar} f
$$


is equal to the Poisson bracket only in the limit $\hbar \rightarrow 0$. Then automorphisms of the Poisson bracket will not be in general automorphisms of the commutator. Nonetheless if the *-product is a function of the Poisson bracket as in the $\mathbb{R}^{2 n}$ case (in that case Weyl quantization furnishes $f * g=\exp \left(\frac{\hbar}{2} \Lambda\right)(d f \wedge d g)$, then any automorphism of the Poisson bracket also preserves the commutator. For our examples $S U(2)$ and $S U(1,1)$ we may use the duality between the algebra of functions, $\mathcal{F}(G)$ on a Lie group and the universal enveloping algebra $U(\mathcal{G})$ of the Lie algebra. Namely, given the product, $\cdot$, on $\mathcal{F}(G)$ and the coproduct $\Delta$ on $U(\mathcal{G})$ we have

$$
<a \cdot b, \xi>=<a \otimes b, \Delta(\xi)>\quad a, b \in \mathcal{F}(G), \quad \xi \in \mathcal{G}
$$

where $\langle a \cdot b, \xi>$ has to be understood as the Lie derivative of $a \cdot b$ with respect to $X_{\xi}$, the realization of $\xi$ on the group; analogously the right hand side is $X_{\xi}(a) X_{\xi}(b)$, where we have used the expression of the coproduct for the generators, namely $\Delta(\xi)=\xi \otimes 1+1 \otimes \xi$. The same relation holds for the deformed algebras [6]:

$$
<a * b, \xi>=<a \otimes b, \Delta_{\hbar}(\xi)>\text {. }
$$

This means that whenever the deformed coproduct $\Delta_{\hbar}$ is invariant, so is the deformed product $*$. For our examples the coproduct is:

$$
\begin{aligned}
\Delta_{\hbar}\left(X^{3}\right) & =X^{3} \otimes 1+1 \otimes X^{3} \\
\Delta_{\hbar}\left(X^{ \pm}\right) & =X^{ \pm} \otimes e^{-\frac{\hbar}{2} X^{3}}+e^{\frac{\hbar}{2} X^{3}} \otimes X^{ \pm}
\end{aligned}
$$

where $X^{ \pm}, X^{3}$ are the generators of the algebra. It can be verified that this coproduct has the same invariance properties as the Poisson bracket defined on the algebra of functions.

These considerations allow the investigation in a quantum setting of dynamical fields which preserve Lie-Poisson structures of $S U(2)$ and $S U(1,1)$. The examples we are going to describe are again only $S U(2)$ and $S U(1,1)$, however we will not consider the quantization problem.

\section{$3.1 S U(2)$}

We use in this example the identification of variables of example (3.1) and we consider the dynamical vector field (41). In order to understand the dynamics we 
Figure 1: The section of the flow diagram for the $S U(2)$ case in the $p_{1}, q_{1}$ plane.

evaluate the equations of the motion which turn out to be

$$
\begin{aligned}
& \dot{q}_{1}=i_{\Gamma} d q_{1}=-q_{1} \\
& \dot{p}_{1}=i_{\Gamma} d p_{1}=-p_{1} \\
& \dot{q}_{2}=i_{\Gamma} d q_{2}=\frac{H_{1}}{H_{2}} q_{2} \\
& \dot{p}_{2}=i_{\Gamma} d p_{2}=\frac{H_{1}}{H_{2}} p_{2}
\end{aligned}
$$

Obviously the Casimirs $\mathcal{C}_{1}, \mathcal{C}_{2}$ are still constants of motion, so that the motion is on the 3 -sphere of constant radius $H_{1}+H_{2}$. It is useful to visualize the motion in the two planes $\left(q_{1}, p_{1}\right)$ and $\left(q_{2}, p_{2}\right)$, which can be considered as the phase spaces of two particles. The motion is described in figures 1 and 2. For both particles (because of the constraint) the motion is confined in the disk of radius $H$, with the center of one circle corresponding to the boundary of the other.

For particle 1 the motion is a contraction to the center, independently of the initial condition, with radial velocity proportional to the radius. The center of the $\left(p_{1} q_{1}\right)$ circle is therefore a sink. By evolving back in time it is easy to see that the boundary of the disk is a source, that is the trajectory of a particle can start from it at a finite time. For the second particle the situation is reversed, the motion is now an expansion, but with a speed which goes to 0 as $t \rightarrow \infty$, so now the center is a source, and boundary a sink. From the global $S^{3}$ point of view we have that the two-sphere $p_{1}^{2}+q_{1}^{2}=1$ is the sink, while the two- sphere $p_{2}^{2}+q_{2}^{2}=1$ is the 
Figure 2: The section of the flow diagram for the $S U(2)$ case in the $p_{2}, q_{2}$ plane.

source. Obviously reversing the sign of the field will change the sink to a source and viceversa.

\section{$3.2 S U(1,1)$}

As we have seen, in the $S U(1,1)$ case there are two inequivalent Lie-Poisson structures which we expect to give rise to different dynamics even in the case we are analyzing. In both situations we consider the dynamical field to be (41), but using the two different identifications of variables (11) and (34). Let us first consider the Lie-Poisson structure defined in (22) and the identifications in (11). The equations of the motion are then

$$
\begin{aligned}
\dot{q}_{1} & =q_{1} \\
\dot{p}_{1} & =p_{1} \\
\dot{q}_{2} & =\frac{H_{1}}{H_{2}} q_{2} \\
\dot{p}_{2} & =\frac{H_{1}}{H_{2}} p_{2}
\end{aligned}
$$


Figure 3: The section of the flow diagram for the $S U(1,1)$ 'boost-boost' case in the $p_{1}, q_{1}$ plane.

This time the motion is on a one-sheeted hyperboloid in $\mathbb{R}^{4}$. We indicate it in figures 3 and 4 depicting the situation, note that while the variables $p_{1}$ and $q_{1}$ can take any value, due to the constraint, $p_{2}$ and $q_{2}$ can only take values outside of the unit circle of the $\left(p_{2}, q_{2}\right)$ plane. The motion of the first particle is a radial expansion, with speed proportional to the radius, while particle 2 has a source on the boundary of its phase space, and then it expands with a speed which increases with time.

For the next case we consider the Lie-Poisson structure

$$
\Lambda=X_{2} \wedge X_{3}-\tilde{X}_{2} \wedge \tilde{X}_{3}
$$

and identify the variables of the phase space as in (34). This time $H_{i}=p_{i}^{2}-q_{i}^{2}$, and $H=H_{1}+H_{2}$. With these choices the dynamical vector field gives the following equations of the motion

$$
\begin{aligned}
\dot{q}_{1} & =-q_{1} \\
\dot{p}_{1} & =-p_{1} \\
\dot{q}_{2} & =\frac{H_{1}}{H_{2}} q_{2} \\
\dot{p}_{2} & =\frac{H_{1}}{H_{2}} p_{2}
\end{aligned}
$$


Figure 4: The section of the flow diagram for the $S U(1,1)$ 'boost-boost' case in the $p_{2}, q_{2}$ plane.

Although apparently similar to the equations in (46) and (47), the phase diagram is actually quite different as can be seen from figure 5 and 6 . In the $\left(q_{1} p_{1}\right)$ plane the motion is a simple contraction with speed proportional to the radius, but in the $\left(q_{2} p_{2}\right)$ plane is rather more complex. The equilater hyperbola $p_{2}^{2}-q_{2}^{2}=1$ corresponds to the origin of the $\left(q_{1} p_{1}\right)$ plane, and is therefore a sink, there are 6 different regions, on some of which the motion is contraction, on some a dilation (as depicted by the arrows in figure 6), the asymptots of the hyperbola are equilibrium positions (they correspond to $H_{1}=0$ ), and the origin is an hyperbolic saddle point.

\section{Conclusions}

The procedure illustrated for the simple cases of $S U(2)$ and $S U(1,1)$ may be generalized to other Lie groups. That is one chooses a Lie-Poisson structure on a Lie group, and investigates the possible Hamiltonian dynamics furnished by the Poisson bracket. This way we are provided with a wide class of non trivial dynamical systems whose phase space is a Lie-Poisson group. This observation enables us to quantize using the deformation procedure which leads to Quantum 
Figure 5: The section of the flow diagram for the $S U(1,1)$ 'boost-rotation' case in the $p_{1}, q_{1}$ plane.

Figure 6: The section of the flow diagram for the $S U(1,1)$ 'boost-rotation' case in the $p_{2}, q_{2}$ plane. 
Groups. In many cases a canonical quantization of the system under consideration is already available, nonetheless there may be systems where this scheme could be a useful one. On the other side there is no standard quantization procedure which allows the investigation of dissipative systems in a quantum setting so that the $*$-deformation tool and quantum groups frame could be useful in the analysis of the quantum meaning of such non conservative dynamics.

\section{AKNOWLEDGEMENTS}

We thank professor W.Thirring and professor P. Michor for their kind hospitality at the Erwin Schroedinger Institut in Wien, where this work was partially done.

\section{REFERENCES}

[1] L. A. Takhtajan in Introduction to Quantum Groups and Integrable Models of Quantum Field Theories, M-L Ge and B-H Zhao eds, World Scientific, Singapore, 1989.

[2] H. D. Doebner, J. D. Henning and W. Lucke in Lecture Notes in Physics 370 $89(19 H)$. D. Doebner and J. D. Hennig eds, Springer Verlag

[3] F. Bayen, M. Flato, C. Fronsdal, A. Lichnerowicz and D. Sternheimer, Deformation Theory and Quantization, Annals of Physics 111 (1978) 61,ibid. 110 (1978) 111.

[4] V. G. Drinfel'd, Hamiltonians structures on Lie groups, Lie bialgebras and the geometric meaning of the classical Yang-Baxter equations Sovieth. Math. Dokl. 27 (1978) 1.

[5] V. Manko, G. Marmo, S. Solimeno, F. Zaccaria, Int. J. Mod. Phys. A (19t) o appear; Phys. Lett. A174B (1993) 173.

[6] T.Tjin An introduction to quantized Lie groups and algebras Amsterdam University Preprint (1991). 